\title{
La aplicación de la ISBD consolidada en la descripción de los recursos electrónicos: similitudes y diferencias con la ISBD(ER)
}

\author{
Ana Belén Ríos HiLARIO y João GUERREIRO \\ Facultad de Traducción y Documentación, Universidad de Salamanca, España \\ anarihi@usal.es
}

\begin{abstract}
Resumen
Se realiza un análisis comparativo entre la ISBD (ER) y la ISBD consolidada centrándonos de manera exclusiva en la descripción de los documentos digitales. El objetivo de nuestro estudio es detectar las semejanzas y disimilitudes entre ambas normas, con la finalidad de proporcionar un resumen de los cambios realizados en la nueva normativa unificada. Tras una introducción en la que se establece la metodología a seguir, se da paso al cotejo de las normas. Dicho examen se divide a su vez en dos partes: la primera, traza la evolución histórica de las dos ISBD, mientras que la segunda establece la comparación propiamente dicha, desde el punto de vista formal y de contenido. Finalmente, en el apartado de las conclusiones se resaltan los aspectos más importantes de la comparación y se señalan una serie de propuestas de mejoras encaminadas a facilitar la aplicación de la ISBD consolidada en lo referente a la descripción de los documentos electrónicos.
\end{abstract}

Palabras clave: Descripción de documentos electrónicos. ISBD (ER). ISBD consolidada.

\section{Introducción}

A estas alturas, describir el proceso de gestión y desarrollo de las ISBD no tiene sentido, sin embargo, permítannos hacer la siguiente reflexión al respecto para sentar las bases del análisis que vamos a efectuar a continuación. La primera ISBD que surge es la dedicada a la descripción de los documentos monográficos, es decir, la ISBD (M). Esto se debe a que la documentación imperante en esas fechas, año 1971, era la monográfica. Años más tarde se establece la ISBD (G) que nace con el objetivo primordial de desarrollar "una descripción bibliográfica internacional normalizada de carácter general que se adecuase a todos los tipos de material bibliotecario" (1993, p. VII). A partir de aquí, proliferaría una serie de ISBD específicas adaptadas a la descripción de los distintos tipos de materiales documentales. La idea estaba clara: esta-

\begin{abstract}
A comparative analysis between the ISBD (ER) and the consolidated ISBD is performed, focusing exclusively on the description of digital documents. The aim of our study is to identify the similarities and differences between the two standards, in order to provide a summary of the changes made in the new uniformed rules. After an introduction which sets out the methodology to follow, the comparison of the rules is carried out. This review is divided into two parts: the first one establishes the historical evolution of both ISBD, while the second one provides the comparison of both form and content. Finally, the conclusions highlight the most important aspects of the comparison and identifies a number of proposals to facilitate the implementation of the consolidated ISBD regarding the description of electronic documents.
\end{abstract}

Keywords: Electronic resources description. ISBD (ER). Consolidated ISBD.

blecer un conjunto de normas que "se fueran adaptando a las distintas necesidades nacionales, pero manteniendo unos mínimos normalizadores que permitiesen un futuro control bibliográfico a nivel universal" (Magán Wals, 2004, p. 186). Sin embargo, tras un periodo de creación de nuevas normas, y revisión de las ya existentes, se plantea la necesidad de desarrollar una nueva normativa unificada, que ve la luz en el año 2007.

Lo que tratábamos de demostrar con esta pequeña reseña histórica es que tras la creación de unas pautas generales se dio paso a la elaboración de una serie de normas de carácter específico, tantas como documentos existían o iban surgiendo, hasta la publicación definitiva de la ISBD consolidadas, en las que se aúna en una única normativa normas generales y específicas. Es decir, en cierto modo hemos vuelto al punto del que partíamos. Ahora nuestra tarea es 
analizar de modo detallado la redacción de esta norma e incidir en las novedades y consecuencias que puede tener la aplicación de la misma, y en lo que a nosotros respecta en la descripción de los documentos digitales.

Partiendo de estas premisas, estableceremos en primer lugar un análisis comparativo entre las anteriores normas de descripción para los recursos electrónicos _ISBD (ER) - y la actual ISBD consolidada. Tal análisis lo haremos en dos niveles: desde el punto de vista histórico y desde el punto de vista constitutivo, centrándonos en la forma y el contenido. Finalmente, en las conclusiones resaltaremos los aspectos más relevantes del estudio, indicando las ventajas y desventajas que la actualización de la normativa tiene a la hora de desarrollar el trabajo de descripción de este tipo de recursos. Destacamos, especialmente la tabla III, en la que hemos querido resaltar los cambios más relevantes y significativos entre una norma y otra y, asimismo, facilitar al lector la comprensión de los mismos.

\section{De la ISBD (ER) a la ISBD Consolidada: análisis diacrónico}

\subsection{ISBD (ER)}

En lo que respecta a los documentos electrónicos, citaremos que las primeras normas que permitían la descripción de este tipo de material fueron las ISBD (NBM) o normas para la descripción del material no librario, publicadas en 1977 y que de modo conjunto trataban la descripción de los documentos audiovisuales, micrográficos, multimedia $\mathrm{y}$, por supuesto, los documentos electrónicos. En 1989 se crea la ISBD (CF) que trata de forma exclusiva el tratamiento de los archivos de ordenador (computer files). Su nacimiento se debe a la celeridad con la que proliferaban, ya por aquel entonces, estos recursos; y su aplicación englobaría a todos los documentos legibles por ordenador independientemente de su presentación, forma de acceso - directo o indirecto- o contenido.

Las sucesivas revisiones de esta norma se debieron a las características inherentes de este tipo de material, entre ellas citaremos: su evolución constante, la sucesión de nuevos soportes y aplicaciones $\mathrm{y}$, fundamentalmente, el avance de las redes

. Y así, se llega al año 1998, cuando esta norma cambia de denominación y se convierte en la ISBD (ER). El cambio en la nomenclatura se produjo, entre otras razones, por las "novedades existentes en el panorama de la edición electrónica y en los problemas para su descripción" (Pedraza Gracia, 1998, p. 50), y del término "archivos" se paso a "recursos" y la expresión "de ordenador" se cambió por el adjetivo de "electrónicos". Sin embargo, este cambio aparentemente formal repercutiría de modo muy significativo en la constitución de la norma, en la composición de las áreas y en la redacción de los elementos que integran las mismas. Aunque estaría bien en este momento establecer una comparación entre la ISBD (CF) y la ISBD (ER), dicho cometido ya fue realizado por varios autores en su momento, y escaparía del objetivo fundamental de nuestra investigación que precisamente es la comparación de la ISBD (ER) con la ISBD consolidada.

La revisión de la ISBD (ER) dio lugar a un borrador en el año 2003 cuya versión definitiva se haya pendiente de aprobación. Ello puede deberse, entre otros motivos, a la redacción y definitiva publicación de las ISBD Consolidadas en el año 2007, y que será objeto de análisis en el siguiente apartado.

\subsection{ISBD Consolidada}

A principio de la década de los noventa se creó el Grupo de Estudio sobre los Requisitos Funcionales para Registros Bibliográficos (FRBR). Se suspendieron la revisión de las ISBD, a excepción precisamente de las dedicadas a los archivos de ordenador, mientras se aguardaba que el citado grupo propusiera un "nivel básico de funcionalidad y unos requisitos básicos de información para los registros creados por las agencias bibliográficas nacionales" (ISBD consolidada, 2008, p. xv).

En 1998 el mencionado grupo publica el informe final y definitivo de los FRBR y con ello se inicia la revisión completa de la familia de las ISBD, a petición del Comité Permanente de la Sección sobre Catalogación de la IFLA, cuyo objetivo principal fue establecer una coherencia entre las estipulaciones de las ISBD y el "nivel básico de registro bibliográfico nacional" propuesto por los FRBR. En el periodo comprendido entre 2000 y 2006 tiene lugar el segundo proyecto de revisión de las ISBD durante el cual se examinan varias normas. Destacamos aquí la aparición en línea de las correspondientes modificaciones de la ISBD (M) y la ISBD (G) en las que por primera vez se estipulan los elementos obligatorios, condicionales y optativos de acuerdo a las recomendaciones de FRBR.

En 2003 el Grupo de Revisión de las ISBD crea el Grupo de Estudios sobre la Dirección Futura de las ISBD, que analizaría la viabilidad de unificar las ISBD específicas, con el propósito de facilitar la descripción de los recursos que comparten características de más de un formato y 
agilizar la labor de mantenimiento y cohesión entre las distintas ISBD.

Un año más tarde, se publica el trabajo de Tom Delsey Mapping ISBD elements to FRBR entity attributes and relationships. Dicha obra tendría como misión encomendada el logro de una adaptación terminológica entre la ISBD y las entidades de los FRBR. Debido al alto nivel de abstracción del modelo conceptual, se optó por mantener la terminología de las ISBD y crear un documento aislado que las relacionase con los FRBR, como sugirió Patrick Le Bouef (2005, p. 82). No obstante, el Grupo de Revisión decidió introducir algunos cambios terminológicos en las ISBD, entre los cuales consta sustituir los términos "ítem", "ejemplar" y "publicación" por el término "recurso".

En 2005 el Grupo de Estudios sobre la Dirección Futura de las ISBD concluye que es viable crear una ISBD consolidada y establece los objetivos y principios que la guiarán publicándose en 2007 el texto preliminar de dicha norma. En el año 2009 se propuso la creación del área 0 (Área de forma del contenido y tipo de medio), que viene a resolver los problemas relativos a la designación general de material encontrados en la edición preliminar de la ISBD. Finalmente, en el año 2010 aparece el borrador de la edición definitiva de la ISBD consolidada, actualmente en fase de discusión.

Aunque hemos abordado el tema histórico de ambas normas por separado, entre otros motivos por cuestiones metodológicas, tenemos que decir que ambas normativas, como todos los textos ISBD, no se desarrollan de forma aislada sino que aunque se redactan mediante la creación de grupos específicos, estos equipos de trabajos no son ajenos al resto de cambios que se producen en el entorno bibliográfico, no sólo en relación con sus propias normas, sino como hemos visto anteriormente, con el resto de componentes que integran el universo catalográfico.

\section{Análisis comparativo de la forma}

La comparación desde un enfoque formal de una norma de carácter general con una norma específica a simple vista puede parecer inapropiada, aparte de que toda la familia ISBD se ha caracterizado por presentar una estructura homogénea, como no podría ser de otra manera, en la estructuración de sus estipulaciones. Sin embargo, hay una serie de apreciaciones formales que creemos oportuno reflejar en este apartado.
En primer lugar y a simple vista, la diferencia en la extensión de ambas normas es notable, evidentemente porque una trata solamente un tipo de recursos, mientras que la Consolidada hacen referencia a todas las tipologías documentales. Este hecho que puede parecer una ventaja también presenta sus inconvenientes. Así, para un descriptor exclusivamente de recursos electrónicos le será más fácil manejar una norma de 109 páginas que otra de 368 , en la que se mezclan las reglas de unos recursos y otros. En este sentido, nos gustaría reflejar que uno de los problemas que presentan la ISBD consolidada es su dificultad de manejo. En relación a este hecho Estivill (2009, p.16) apunta que

la ISBD consolidada presenta deficiencias de organización que dificultan la localización de las reglas que se aplican a materiales específicos, ya que el sumario es muy general y el índice no incluye entradas ni subentradas para estas reglas específicas.

Por lo tanto, desde aquí abogamos por una reestructuración de la norma que solucione los problemas de comprensión y localización de los distintos elementos que componen la misma.

Si nos fijamos en la estructura, la primera ISBD (ER) consta de una introducción, un capítulo denominado notas preliminares, la especificación de las distintas áreas distribuidas en ocho capítulos, uno para cada área, cinco apéndices y un índice. Idéntica estructura presenta la Consolidada. Tras una introducción, se organiza la información en un capítulo preliminar, ocho capítulos centrales, uno para cada una de las áreas de la descripción bibliográfica, cinco apéndices y un índice. En el capítulo preliminar se dan una serie de diferencias entre ambas normas. Así, la ISBD (ER) el segundo y tercer apartado hace referencia a "Definiciones" y "Esquema comparativo entre de la ISBD (G) y la ISBD (ER)", mientras que en la Consolidada desaparecen estos dos puntos y en su lugar, el segundo apartado hace referencia a "Tratamiento de los recursos". Por su parte, esta norma incluye un último apartado denominado "Imperfecciones" que no está presente en la ISBD (ER).

Por otro lado diremos que el apartado destinado a los ejemplos -que figura en un apéndice en la ISBD (ER) - se presenta como un suplemento publicado de modo independiente en el caso de la ISBD consolidada.

La publicación de la ISBD (ER) se realizó en formato impreso por la editorial K. G. Saur y se encuentra disponible en línea en formato HTML. La revisión y actualización de esta norma, pendiente de aprobación, se encuentra disponible 
en formato PDF. Por su parte, la versión impresa de la ISBD consolidada se publicó por la misma editorial y figura en línea en formato PDF. La traducción al español de esta última norma fue realizada por la Biblioteca Nacional y está disponible en línea en formato PDF en la web de la citada institución.

\section{Análisis del contenido}

\subsection{Definición}

Para llevar a cabo este análisis del contenido, en primer lugar, comenzaremos indicando qué entienden estas normas por la categoría documental de "recurso electrónico". La ISBD (ER) define el término en el apartado "0.1.1 Alcance" mientras que la Consolidada lo detalla en dos momentos: en el apartado "0.2.1 Tipos específicos de recursos" y en el "Glosario" que figura en uno de los apéndices. En ambas normas se proporciona idéntica información, lo que sucede es que mientras que la primera de las normas incluye toda la definición en único apartado, la ISBD consolidada destina el Glosario para definir recurso electrónico de modo general, y el apartado 0.2.1 para precisar el término desde el punto de vista catalográfico. Para ilustrar dicho concepto hemos recogido la definición genérica que figura en el Glosario de la ISBD consolidada sobre recurso electrónico (ISBD consolidada, 2008, p. 351):

Recurso formado por materiales controlados por ordenador, incluyendo los materiales que requieren el empleo de un periférico (ej. un lector de CDROM) conectado a un ordenador; los recursos pueden usarse en modo interactivo o no. Se incluyen dos tipos de recursos: los de datos (información en forma numérica, alfabética, gráfica, de imágenes y sonido o una combinación de todo lo anterior) y los de programas (instrucciones o rutinas para ejecutar ciertas tareas que incluyen el proceso de datos). Además, pueden combinarse e incluir datos electrónicos y programas (ej. software educativo con texto, gráficos y programas).

Esta definición de recurso electrónico se aplica a la mayor parte de los recursos generalmente disponibles e incluye aquellos recursos accesibles mediante telecomunicaciones. También comprende los recursos producidos o generados para su distribución limitada, pago bajo demanda o por encargo. Un recurso que reside en la memoria permanente de un ordenador (ROM) se entiende como parte del dispositivo en el que se almacena; y, si se cataloga, se trata como un recurso que requiere un acceso remoto. Los juegos de ordenador, calculadoras y otros objetos programados están fuera del ámbito de la ISBD.

\subsection{Notas preliminares}

En el primer apartado de ambas normas, denominado "Alcance, objetivo, uso" existe una diferenciación en el modo de clasificar los elementos. Así, mientras en la ISBD (ER) los elementos son obligatorios $\mathrm{u}$ opcionales, la Consolidada define un nuevo tipo denominado condicional. A continuación recogemos las definiciones que de tales conceptos realiza la norma unificada (2008, p. II):

- Obligatorio: El elemento se requiere en todas las situaciones en las que sea aplicable; se indica en el texto por "se da" o "se dan".

- Condicional: El elemento se requiere en determinadas condiciones, tales como "cuando sea necesario para la identificación o se considere importante para los usuarios del catálogo". Si la condición no se cumple, el uso del elemento es opcional.

- Opcional: De puede o no incluir el elemento, a discreción de la agencia, lo que se indica en el texto por "opcional" o "puede".

Precisamente, la inclusión de estos tipos se debe a la aplicación del modelo FRBR en el desarrollo de la norma. Sin embargo, aprovechamos la ocasión para comentar que una de las críticas que recibe la nueva normativa, es la poca influencia que dicho modelo tiene en la redacción de la nueva normativa.

En el apartado destinado a la puntación, existe una diferencia notable en la nueva normativa con respecto al resto de las ISBD, no sólo a la referente a los recursos electrónicos. En lo que se refiere a la regla 0.3.2.7 (ISBD consolidada, 2007, p. XXI):

Si un elemento o área termina con un punto y la puntuación prescrita para el elemento o área que le sigue comienza con un punto, se dan ambos puntos, con el fin de tener en cuenta tanto la puntuación prescrita como la de las abreviaturas.

\begin{tabular}{ll}
\hline$I S B D$ & ISBD consolidada \\
\hline 3rd ed. - & 3rd ed.. - \\
\hline
\end{tabular}

Tabla I. Puntuación final

Por su parte, el punto 0.3.2.8 realiza un cambio importante en el empleo de los corchetes (ISBD consolidada, 2008, p. XXII):

Cuando sucesivos elementos dentro de una misma área se obtienen fuera de la fuente de información prescrita, cada elemento se encierra en su propio par de corchetes. 
Las normas anteriores decían que dicha información debía consignarse en un único par de corchetes.

\begin{tabular}{ll}
\hline$I S B D$ & $I S B D$ consolidada \\
\hline.$-[$ S.I. : s.n.] & . - [S.I.] : [s.n.] \\
\hline
\end{tabular}

Tabla II. Uso de los corchetes

El último de los cambios referentes a la puntuación es el siguiente (ISBD consolidada, 2008, p. XXII):

Cuando una única mención (ej. una mención de responsabilidad, véase 1.5) se da en parte en una lengua $y / o$ escritura $y$, en parte, en más de una lengua y/o escritura, las diversas formas lingüísticas se transcriben juntas; se utilizan el signo igual y otros símbolos de puntuación, según sea apropiado.

El objetivo de dichos cambios obedece a razones de interoperabilidad entre los diferentes sistemas de recuperación y los formatos de presentación.

\section{3. Áreas}

En el área de título y mención de responsabilidad (área 1) se aprecian varios cambios. La primera diferencia respecto a esta área, según la Consolidada, hace referencia a que el título paralelo se considera un elemento condicional mientras que en la ISBD (ER) era un elemento obligatorio. Lo mismo ocurre con la información complementaria que pasa de ser imprescindible a tener un carácter condicional. Respecto a los responsables que deben consignarse en esta área, la ISBD específica no establecía ninguna distinción desde el punto de vista de la responsabilidad, mientras que las nuevas normas pasan a diferenciar entre menciones de responsabilidad principales y secundarias. En relación con este aspecto, la mención de responsabilidad era un elemento obligatorio en la ISBD (ER), mientras que la Consolidada establece como obligatoria la responsabilidad principal y como condicional el resto de responsabilidades.

En el área de edición (área 2) no se establece ninguna diferencia entre ambas normas.

Respecto al área de designación y extensión del recurso (área 3), en la primera ISBD específica de los recursos electrónicos se definió esta área como "Designación y extensión del recurso" y en ella se consignaba la información referente a estos dos elementos. Posteriormente, en la revisión que se hizo de las ISBD se decidió la omisión de esta área debido a que no ofrecía ninguna información relevante a la hora de rea- lizar la descripción y posterior recuperación del documento.

En el área de publicación, producción, distribución, etc. (área 4), se ha producido un cambio en la denominación de la misma, que ha pasado de llamarse "área de publicación, distribución, etc." a incluir el término de producción en medio de ambos términos.

Por otro lado, ha habido una variación respecto al tratamiento que se le otorga al editor, productor o distribuidor, puesto que deberá consignarse siempre que se conozca su nombre, aunque éste haya sido extraído de una fuente externa al propio documento. En nota se consignará la fuente de la que hemos tomado este dato.

La primera diferencia en el área de descripción física (área 5) es la que específica las fuentes de información para la descripción de la misma. Mientras que en la ISBD (ER) establece cualquier fuente en la Consolidada hace referencia a "el recurso en su totalidad". Otro cambio es que mientras en la primera norma no se considera la extensión del recurso ya que esta información figuraba en el área 3 , en la ISBD consolidada existe un apartado para constatar este dato.

En el área de serie (área 6), la diferencia entre ambas normas radica en la definición de las fuentes. Si la ISBD (ER) determinaba el recurso en sí mismo, la Consolidada determina, en primer lugar, una diferenciación entre los recursos de acceso directo y los recursos de acceso remoto.

En el área de notas (área 7) podemos reseñar como cambio primordial la estipulación que figura en la ISBD consolida, que dispone que "cuando una nota es obligatoria, pero el catalogador no conoce la información necesaria para redactarla, se indica esa circunstancia en nota" (ISBD consolidada, 2008, p. 253).

Finalmente, en el área de identificador del recurso y condiciones de disponibilidad (área 8), de nuevo la diferencia entre ambas normas viene marcada por la categorización de los elementos. Así, mientras que en la ISBD (ER) el número normalizado era obligatorio, en la Consolidada pasa a ser condicional.

\subsection{Apéndices}

En cuanto a los apéndices, excepto el E, la denominación de los mismos es igual lo único que varía es su contenido. Este último apéndice se destina en el caso de las ISBD (ER) a una relación de ejemplos, mientras que en la ISBD consolidada se destina al Glosario. 


\begin{tabular}{|c|c|c|c|}
\hline Sección & & ISBD (ER) & ISBD consolidada \\
\hline \multirow{12}{*}{$\begin{array}{l}\text { Notas } \\
\text { preliminares }\end{array}$} & 0.1 & Alcance, objetivo, uso & Alcance, objetivo, uso \\
\hline & & $\begin{array}{l}\text { Presenta dos tipos de elementos: obligatorio y } \\
\text { opcional }\end{array}$ & $\begin{array}{l}\text { Presenta tres tipos de elementos: obligatorio, } \\
\text { condicional y opcional }\end{array}$ \\
\hline & 0.2 & Definiciones & Tratamiento de los recursos \\
\hline & 0.3 & $\begin{array}{l}\text { Esquema comparativo de la ISBD (G) y la } \\
\text { ISBD (ER) }\end{array}$ & Esquema de la ISBD y puntuación \\
\hline & 0.4 & Puntuación & Fuentes de información \\
\hline & 0.5 & Fuentes de información & Lengua y escritura de la descripción \\
\hline & 0.6 & Lengua y escritura de la descripción & Omisiones y abreviaturas \\
\hline & 0.7 & Omisiones y abreviaturas & Uso de mayúsculas \\
\hline & 0.8 & Uso de mayúsculas & Ejemplos \\
\hline & 0.9 & Ejemplos & Erratas \\
\hline & 0.10 & Erratas & Símbolos \\
\hline & 0.11 & Símbolos & Imperfecciones \\
\hline \multirow[t]{5}{*}{ Área 1} & & El título paralelo es un elemento obligatorio & El título paralelo es un elemento condicional \\
\hline & & $\begin{array}{l}\text { El complemento de título es un elemento } \\
\text { obligatorio }\end{array}$ & El complemento de título es un elemento condicional \\
\hline & & No hace diferencia entre las menciones de & principal y secundaria \\
\hline & & responsabilidad & La mención de responsabilidad principal es \\
\hline & & $\begin{array}{l}\text { La mención de responsabilidad es un } \\
\text { elemento obligatorio }\end{array}$ & $\begin{array}{l}\text { obligatoria, mientras que la secundaria es un } \\
\text { elemento condicional }\end{array}$ \\
\hline \multicolumn{4}{|l|}{ Área 2} \\
\hline Área 3 & & $\begin{array}{l}\text { Desaparece (No se aplica a los recursos } \\
\text { electrónicos) }\end{array}$ & \\
\hline \multirow[t]{2}{*}{ Área 4} & & $\begin{array}{l}\text { Denominación: Publicación, distribución, etc. } \\
\text { No figura en la ER }\end{array}$ & $\begin{array}{l}\text { Denominación: Publicación, producción, distribución, } \\
\text { etc. }\end{array}$ \\
\hline & & & $\begin{array}{l}\text { 4.2.11 Cuando el nombre del editor, productor o } \\
\text { distribuidor se obtiene de una fuente de información } \\
\text { distinta a la prescrita para el recurso (incluyendo } \\
\text { fuentes externas a éste), se proporciona entre } \\
\text { corchetes y en la forma apropiada a la fecha, } \\
\text { escritura y lengua del recurso. Esta fuente de } \\
\text { información se registra en el área } 7 \text {. }\end{array}$ \\
\hline \multirow[t]{2}{*}{ Área 5} & & Fuente de información: cualquier fuente & Fuente de información: Recurso en su totalidad \\
\hline & & $\begin{array}{l}\text { No se considera la extensión de los recursos } \\
\text { electrónicos }\end{array}$ & $\begin{array}{l}\text { Existe un apartado para la extensión del recurso } \\
\text { (dado en bytes, kilobytes) }\end{array}$ \\
\hline \multirow[t]{3}{*}{ Área 6} & & Fuente de información: recurso en sí mismo & Fuente de información: \\
\hline & & (documentación, otro material y envase) & Acceso directo: el recurso en sí mismo \\
\hline & & & $\begin{array}{l}\text { Acceso remoto: Pantalla de título, menú principal, } \\
\text { menciones del programa, etc. }\end{array}$ \\
\hline Área 7 & & - & $\begin{array}{l}\text { Cuando una nota es obligatoria, pero no se conoce la } \\
\text { información necesaria, se indica esa circunstancia en } \\
\text { nota. }\end{array}$ \\
\hline Área 8 & & $\begin{array}{l}\text { El número normalizado es un elemento } \\
\text { obligatorio }\end{array}$ & El número normalizado es un elemento condicional \\
\hline \multirow[t]{4}{*}{ Apéndices } & & Apéndice A: Descripción a varios niveles & Apéndice A: Descripción a varios niveles \\
\hline & & Apéndice B: Registros bidireccionales & Apéndice B: Registros bidireccionales \\
\hline & & $\begin{array}{l}\text { Apéndice C: Designaciones generales y } \\
\text { especificas de material }\end{array}$ & $\begin{array}{l}\text { Apéndice C: Designaciones generales y específicas } \\
\text { de material }\end{array}$ \\
\hline & & $\begin{array}{l}\text { Apéndice D: Abreviaturas } \\
\text { Apéndice E: Ejemplos }\end{array}$ & $\begin{array}{l}\text { Apéndice D: Abreviaturas } \\
\text { Apéndice E: Glosario }\end{array}$ \\
\hline
\end{tabular}

Tabla III. De la ISBD (ER) a la ISBD consolidada: principales cambios en la descripción de los recursos electrónicos similitudes y diferencias con la ISBD (ER). // Scire. 17:1 (en.-jun. 2011) 41-48. ISSN 1135-3716. 


\section{Conclusiones}

En lugar de dedicar este espacio de forma única a resaltar los aspectos más importantes que hemos obtenido en la comparación de ambas normas, vamos a realizar una serie de propuestas encaminadas a la mejora de la norma consolidada en lo que respecta a la aplicación de la misma a la descripción de los recursos electrónicos.

En primer lugar, creemos que sería muy positiva una revisión de la organización actual de la norma en aras a facilitar la búsqueda de las distintas reglas. La actual presentación resulta muy engorrosa sobre todo en aquellos apartados donde el tratamiento de las distintas provisiones es muy diferente según el tipo de recurso que se trate.

Por otro lado, la posible influencia del esquema FRBR sólo queda reflejado en la ISBD consolidada a través de la clasificación de los elementos en obligatorios, condicionales y optativos.

Hay un apartado de la norma que no hemos mencionado en el análisis, al no estar directamente relacionado con la descripción de los recursos electrónicos, objeto de nuestro estudio; pero que nos parece interesante apuntar en este momento. No existe una correspondencia entre los tipos de recursos definidos en la norma y el resto de ISBD específicas. Por ejemplo, en dicha enumeración se echan en falta los recursos continuados, objeto de las ISBD (CR). En este sentido, Estevill (2009, p. 15) señala que

a partir de la sección 0.2 y hasta el final del texto se van dando instrucciones específicas para el tratamiento de tipos concretos de recursos, en las que se mezclan las instrucciones para aquellos materiales (por ejemplo, recursos electrónicos, imágenes en movimiento) con instrucciones sobre los diversos tipos de recursos continuados. Se trata básicamente de una falta de consistencia que habrá que solucionar en la edición definitiva del texto.

Otra cuestión que no podemos omitir es el hecho de que se ha aprobado la creación una nueva área denominada área 0 , "Área de Forma del Contenido y de Tipo de Medio", que sustituye al elemento "Designación general de la clase de material". Dicho elemento tal y como estaba definido resultaba incoherente al mezclar información sobre el contenido con información sobre el soporte, lo cual resultaba contradictorio. En este sentido, estamos a la espera de la aprobación definitiva de la revisión de la ISBD consolidada que ya incluye en su estructura esta nueva área. En el futuro veremos de qué manera puede influir en la descripción de los documentos digitales este hecho.

No quisiéramos terminar este apartado sin resaltar todos aquellos aspectos positivos que la ISBD consolidada presenta en relación a la descripción de los documentos electrónicos.

Las pautas son más claras, lo que facilita su comprensión por parte del catalogador. De las pautas generales para todo tipo de material, se pasan a definir las normas específicas según el tipo de recurso. En este sentido, y por sus características inherentes, las especificaciones para los documentos digitales tienen un peso importante en el conjunto de la norma. Por otro lado, esta estructura resulta muy útil para la catalogación de aquellos materiales que comparten características de varias ISBD, lo que ocurre con bastante frecuencia en los recursos electrónicos.

Destacamos los cambios referentes a la puntuación que tienen como objetivo facilitar la interoperatibilidad entre formatos y sistemas. También es muy importante el Glosario que figura al final del texto que ayuda a la comprensión de la terminología empleada en el mismo.

La Biblioteca Nacional de España ha establecido que se sigan estas pautas en la descripción de los documentos digitales, en lugar del capítulo 11 de las Reglas de Catalogación españolas dedicadas a los "Archivos de ordenador".

Finalmente, nos gustaría destacar el gran esfuerzo que están llevando a cabo, tanto los organismos internacionales como nacionales, para adecuar la normativa a los cambios que se están produciendo de modo vertiginoso en el universo bibliográfico. En este sentido, actualmente la ISBD consolidada "no solucionará todos los problemas que están presentes hoy en día en los procesos de catalogación" (2008, p. xvi). Sin embargo, el Grupo de Revisión es consciente, tal y como declara en la introducción de la norma, que se puede alcanzar una mayor coherencia y uniformidad. Así, dicho grupo está trabajando en una nueva revisión encaminada a conseguir tal propósito y resolver algunos problemas remanentes, teniendo en consideración otras propuestas procedentes de todas las Reuniones IFLA de Expertos sobre un Código Internacional de Catalogación (IME-ICCs).

\section{Referencias}

Delsey, Tom (2004). Mapping ISBD elements to FRBR entity attributes and relationships. [S.I]: IFLA, 2004. http://archive.ifla.org/VII/s13/pubs/ISBD-FRBR-mapping Final.pdf (2011-04-08)

Estivill Rius, Assunpció (2009). Estado actual de la normativa de catalogación. $1^{\text {a }}$ parte: el escenario internacional. //

Ríos Hilario, Ana Belén; Guerreiro, João. La aplicación de la ISBD consolidada en la descripción de los recursos electrónicos: similitudes y diferencias con la ISBD (ER). // Scire. 17:1 (en.-jun. 2011) 41-48. ISSN 1135-3716. 
Bid: textos universatis de biblioteconomía i documentació. ISSN 1575-5886. 22 (Jun. 2009). http://diposit. ub.edu/dspace/bitstream/2445/16821/1/571855esp.pdf (2011-04-13).

Escolano Rodríguez, Elena; McGarry, Dorothy (2007). ISBD consolidada: um passo em frente. [Lisboa]: Biblioteca de Arte da Fundação Caloust Gulbenkien, 2007. http://imeicc5.com/download/portuguese/ISBD\%20Portu guese.pdf (2011-04-13)

Full ISBD Examples: Supplement to the preliminary consolidated edition of the International Standard Bibliographic Description (ISBD). Preliminary edition. La Haya: International Federation of Library Associations and Institutions, ISBD Review Group, 2009. http://www.ifla.org/files/cataloguing/isbd/isbd-examples_ 2009.pdf (2011-04-22)

Guerreiro, João (2010). De la ISBD (ER) a la ISBD (Consolidada): análisis de la normativa para la descripción de los documentos digitales. Salamanca: Universidad de Salamanca, 2010. http://gredos.usal.es/jspui/handle/10366/ 83113 (2011-04-11). Trabajo de final de máster.

ISBD (ER). International Standard Bibliographic Description for Electronic Resources. Munich: K. G. Saur, 1997. http://archive.ifla.org/VII/s13/pubs/isbd.htm (2011-0421).

ISBD (ER). International Standard Bibliographic Description for Electronic Resources: Draft for World Wide Revision.
IFLA, 2004. http://www.ifla.org/VII/s13/guide/isbder_ ww2-1-04.pdf ( 2011-04-21)

ISBD (G): Descripción bibliográfica internacional normalizada general. Madrid: ANABAD: ARCOS LIBROS, 1993.

ISBD (M): Descripción bibliográfica internacional normalizada para publicaciones monográficas. Madrid: ANABAD: ARCOS LIBROS, 1993.

(ISBD): Descripción Bibliográfica Internacional Normalizada (2008). Ed. Preliminar consolidado. [S.I]: IFLA; [Madrid]: Biblioteca Nacional de España, 2008. http://www.bne. es/es/Servicios/NormasEstandares/ISBDconsolidada/Do cs/ISBDconsolidada.pdf (2011-04-13)

Le Boeuf, Patrick (2005). El mundo feliz de los FRBR. // Principios de Catalogación de IFLA: pasos hacia un Código Internacional de Catalogación: informe de la $1^{\text {a }}$ Reunión IFLA de Expertos sobre un Código Internacional de Catalogación, Frankfurt, 2003. [Madrid]: Secretaria General Técnica, Subdirección General de Publicaciones, Información y Documentación, 2005. 80-90.

Margán Wals, José Antonio (coordinador) (1995). Tratado básico de biblioteconomía. Madrid: Editorial Complutense, 1995. ISBN: 8489365148

Pedraza Gracia, Manuel José. Las ISBD (ER): adecuación normativa a un panorama cambiante. // Scire. ISSN: 1135-3716. 4:1 (en.-jun. 1998) 45-55. 